\title{
Papillomatosis cutis lymphostatica: a sign of chronic edema
}

\section{Mariem Daldoul', Amina Aounallah', Sana Mokni', Taghrid Tlili², Badreddine Sriha², Colandane Belajouza', Mohamed Denguezli ${ }^{1}$}

\author{
${ }^{1}$ Department of Dermatology, Farhat Hached University Hospital, Sousse, Tunisia, ${ }^{2}$ Department of Pathology, Farhat
} Hached University Hospital, Sousse, Tunisia

Corresponding author: Dr. Mariem Daldoul, E-mail: myriam-dal@hotmail.com

\begin{abstract}
An obese 53-year-old male presented himself to our department with lymphedema of the left leg and ulcerated lesions evolving for two weeks prior. His medical history included epilepsy since childhood, mental retardation, chronic venous insufficiency, and chronic lymphedema complicated by recurrent ulceration and cellulitis. Over the previous four years, he had developed verrucous and papillomatous lesions on the left leg. A physical examination revealed an edematous leg with multiple painless coalescent verrucous skin-colored papules with a smooth or hyperkeratotic surface on the left lower leg surrounding the ulceration. The lesions were associated with oozing. A skin biopsy revealed hyperkeratosis, verrucoid acanthosis, and papillomatosis of the epidermis with moderate perivascular inflammatory infiltration of the dermis. A diagnosis of papillomatosis cutis lymphostatica (PCL) was reached based on histopathological and clinical findings. Our patient received lymphatic drainage and locally $5 \%$ salicylic acid.
\end{abstract}

Key words: Chronic lymphedema; Papillomatosis cutis lymphostatica; Elephantiasis nostras verrucosa

\section{INTRODUCTION}

Papillomatosis cutis lymphostatica (PCL), or elephantiasis nostras verrucosa, is a rare, benign, and asymptomatic condition affecting usually the lower legs and resulting from chronic lymphedema [1]. Herein, we report a new sporadic case of PCL.

\section{CASE REPORT}

An obese 53-year-old male presented himself to our department with lymphedema of the left leg and ulcerated lesions evolving for two weeks prior. His medical history included epilepsy since childhood, mental retardation, chronic venous insufficiency, and chronic lymphedema complicated by recurrent ulceration and cellulitis. Over the previous four years, he had developed verrucous and papillomatous lesions on the left leg. A physical examination revealed an edematous leg with multiple painless coalescent verrucous skin-colored papules with a smooth or hyperkeratotic surface on the left lower leg surrounding an ulceration $10 \times 5 \mathrm{~cm}$ in size (Fig. 1). The lesions were associated with oozing. A skin biopsy revealed hyperkeratosis, verrucoid acanthosis, and papillomatosis of the epidermis with moderate perivascular inflammatory infiltration of the dermis (Fig. 2). A diagnosis of PCL was reached based on histopathological and clinical findings. The patient received lymphatic drainage and locally $5 \%$ salicylic acid.

\section{DISCUSSION}

Normally, PCL results from primary or secondary lymphedema or from damage to lymphatic vessels by metabolic diseases, chronic infections, or venous insufficiency [1]. Obstruction of lymphatic drainage causes an excess of protein in the affected regions, inducing fibroblast proliferation and increasing

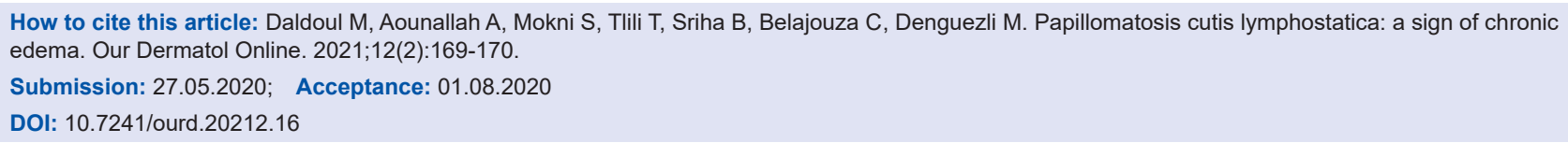




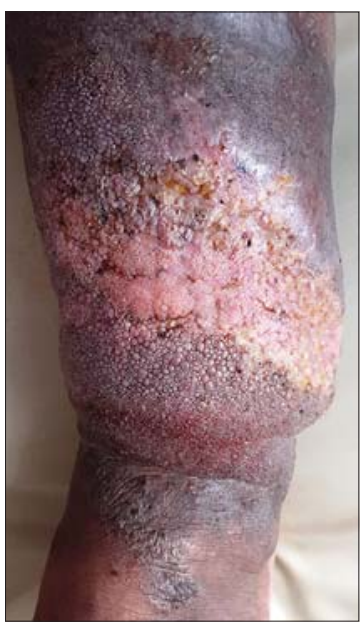

Figure 1: A papillomatous skin-colored lesion on the left leg.

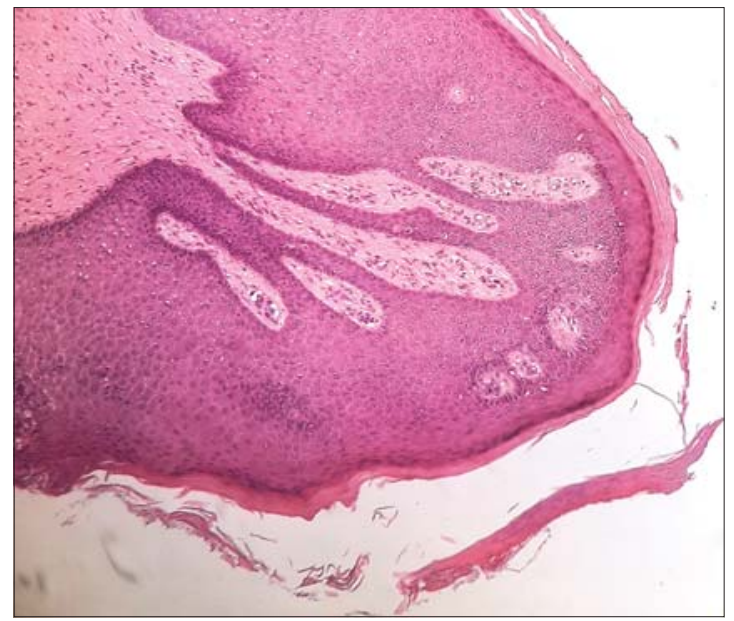

Figure 2: A biopsy of the papillomatous lesion showing hyperkeratosis, verrucoid acanthosis, and papillomatosis of the epidermis.

the recurrence of infections [2]. Also, alteration of the lymph flow leads to the formation of dilated and congested dermal collaterals. The most typical locations are the legs, feet, toes, and around venous ulcers.

The use of compression stockings is the cornerstone of conservative management. Topical and oral retinoids have shown therapeutic efficacy in many cases reported by interfering with epidermal proliferation and inflammation [3-5].

Topical keratolytic agents can be an option. Surgical interventions and $\mathrm{CO}_{2}$ laser excision might be performed if other treatments fail.

\section{CONCLUSION}

As far as we know, few cases of PCL have been reported, and PCL remains a vulnerable area in the occurrence of infection and neoplasia. We insist on the importance of its early recognition to prevent complications.

\section{Consent}

The examination of the patient was conducted according to the principles of the Declaration of Helsinki.

The authors certify that they have obtained all appropriate patient consent forms, in which the patients gave their consent for images and other clinical information to be included in the journal. The patients understand that their names and initials will not be published and due effort will be made to conceal their identity, but that anonymity cannot be guaranteed.

\section{REFERENCES}

1. Kasper RS, Nobbe S. Images in clinical medicine. Papillomatosis cutis lymphostatica. N Engl J Med. 2014;370:69.

2. Sisto K, Khachemoune A. Elephantiasis nostras verrucosa: a review. Am J Clin Dermatol. 2008;9:141-6.

3. Marasca C, Mascolo M, Ferrillo M, Masarà A, Annunziata MC, Iacobelli A, et al. Acitretin may improve symptoms and exudation in patients affected by elephantiasis nostras verrucosa: report of a case. Int Wound J. 2019;16:579-81.

4. Polat M, Sereflican B. A case of elephantiasis nostras verrucosa treated by acitretin. J Drugs Dermatol. 2012;11:402-5.

5. Feind-Koopmans A, van de Kerkhof PC. Successful treatment of papillomatosis cutis lymphostatica with acitretin. Acta Derm Venereol. 1995;75:411.

Copyright by Mariem Daldoul, et al. This is an open-access article distributed under the terms of the Creative Commons Attribution License, which permits unrestricted use, distribution, and reproduction in any medium, provided the original author and source are credited. Source of Support: Nil, Conflict of Interest: None declared. 